\title{
Balkanologie
}

Balkanologie Revue d'études pluridisciplinaires

Vol. IV, $n^{\circ} 2 \mid 2000$

Volume IV Numéro 2

\section{Nationalisme et terrorisme en Macédoine vers 1900}

\section{Nadine Lange-Akhund}

\section{OpenEdition}

Journals

Édition électronique

URL : http://journals.openedition.org/balkanologie/320

DOI : 10.4000/balkanologie.320

ISSN : 1965-0582

\section{Éditeur}

Association française d'études sur les Balkans (Afebalk)

\section{Édition imprimée}

Date de publication : 1 décembre 2000

ISSN : 1279-7952

\section{Référence électronique}

Nadine Lange-Akhund, « Nationalisme et terrorisme en Macédoine vers 1900 », Balkanologie [En ligne], Vol. IV, n 2 | 2000, mis en ligne le 20 juillet 2011, consulté le 17 décembre 2020. URL : http:// journals.openedition.org/balkanologie/320; DOI : https://doi.org/10.4000/balkanologie.320

Ce document a été généré automatiquement le 17 décembre 2020.

(C) Tous droits réservés 


\title{
Nationalisme et terrorisme en Macédoine vers 1900
}

\author{
Nadine Lange-Akhund
}

1 Vers 1900, la Macédoine était l'un des derniers territoires de la péninsule balkanique qui n'avait pas encore réussi à obtenir l'autonomie ou l'indépendance de la part des Ottomans ${ }^{1}$. Accompagnant le déclin de l'empire ottoman, l'idée de combattre le "joug" $\mathrm{du}$ sultan pour construire un État-nation libre et indépendant selon l'un des principes de la révolution de 1848, le droit des peuples à disposer d'eux-mêmes, avait déjà fait son chemin parmi les mentalités balkaniques. Dès cette époque, la Macédoine aurait-elle, elle aussi, pu devenir un de ces nouveaux États émergeant du retrait graduel de la présence séculaire des Turcs en Europe ${ }^{2}$ ?

2 En réalité, la Macédoine se résumait à une simple expression géographique, centrée sur la vallée du Vardar, l'axe de communication nord-sud reliant Belgrade et Salonique. La Macédoine était divisée en trois secteurs administratifs ottomans ou vilayets, le plus vaste étant celui de Salonique, suivi par celui de Bitola à l'ouest vers l'Albanie. Le vilayet de Kosovo s'étendait au nord vers la Serbie.

3 Les populations, moins de trois millions d'habitants, étaient extrêmement mélangées, comprenant des groupes bulgares, serbes, grecs, valaques, tsiganes, turques et albanais, dont aucun ne formait la majorité. De façon générale, les campagnes étaient peuplées par des Slaves. La plupart des paysans bulgares ou serbes vivaient sur des grands domaines ottomans. Les villes et les bourgs rassemblaient des populations grecques et turques. À Salonique, une large communauté juive formait environ la moitié de la population de la ville ${ }^{3}$. Le sentiment de conscience nationale macédonienne était pratiquement inexistant, parce que ces populations s'identifiaient d'abord en fonction de leur appartenance religieuse, orthodoxe, musulmane ou juive. Par conséquent, la Macédoine était devenue un champ libre où rivalisaient les ambitions politiques des États voisins, la Grèce, la Bulgarie et la Serbie, dont les gouvernements respectifs considéraient que "leurs frères de race" devaient être libérés de gré ou de force de la domination ottomane. Athènes, Sofia et Belgrade souhaitaient élargir leurs frontières respectives en y incluant une large portion des trois vilayets macédoniens. 
Vers 1900, ces trois gouvernements soutenaient plusieurs mouvements de libération nationale qui combattaient pour libérer la Macédoine des Ottomans avec une violence qui surprenait et choquait parfois l'opinion publique européenne.

\section{Les origines des mouvements de libération nationale}

5 Quatre mouvements de libération sont ici à considérer, trois d'entre eux ont été fondés hors de Macédoine et un seul s'est établi en tant que mouvement national macédonien. Ils font leur apparition sur la scène macédonienne durant les années 1890. Les mouvements bulgare et grec peuvent être présentés ensemble en raison de la similitude de leur organisation.

6 En Bulgarie, une importante communauté macédonienne vit à Sofia, car il n'y a aucun futur à envisager pour ces chrétiens orthodoxes en Macédoine. Avocats, médecins, fonctionnaires, officiers dans l'armée bulgare, ils tentent d'influencer le gouvernement bulgare en faveur de la cause macédonienne. Depuis le début des années 1890, ces émigrés ont fondé des associations culturelles, publient des revues défendant les intérêts de "leurs frères" de Macédoine, et organisent des manifestations de soutien. Parallèlement des officiers ont formé des ligues militaires, car ils envisagent de mener une lutte armée en Macédoine. Nombreux sont ceux qui estiment que la population des trois vilayets est, en fait, bulgare et, par conséquent, que ces terres doivent être rattachées à la Bulgarie.

7 En 1895, un mouvement bulgare organisé émerge d'un congrès général des différentes associations tenu à Sofia, le Comité Suprême Macédonien, dirigé par le général Danail Nikolaev, un militaire de carrière qui a participé à l'annexion de la Roumélie Orientale en $1885^{4}$. En 1901, un autre militaire, le général Ivan Cončev, s'impose à la direction du Comité Suprême. De caractère fort, il jouit d'un certain prestige auprès des officiers. Proche du milieu de la cour, il bénéficie de l'appui du Prince Ferdinand de SaxeCobourg-Gotha. Ce dernier, au pouvoir depuis 1887, a pour ambition secrète de reconstituer la "grande Bulgarie" et soutient discrètement le mouvement par un appui financier.

8 Le Comité Suprême Macédonien a été créé dans des conditions favorables à son développement, bénéficiant d'une large liberté d'organisation et d'action, ainsi que de la sympathie de l'opinion publique bulgare, dont le pays a été récemment libéré de la tutelle ottomane.

9 Comme en Bulgarie, il existe en Grèce de nombreuses associations de défense de la cause grecque en Macédoine. Ces groupes demandent le rattachement de la province à la Grèce et essayent de gagner l'opinion publique à leurs idées. Les convictions des Grecs trouvent leurs origines dans la "Megali Idhea", un courant de pensée datant du mouvement de la libération qui avait précédé l'indépendance de la Grèce en 1830. La "Megali Idhea", c'est-à-dire "grande idée", rencontre toujours un écho favorable parmi les cercles militaires et intellectuels d'Athènes. Son but est de rassembler tous les Grecs se trouvant hors du royaume, ainsi que leurs terres afin de recréer l'ancien empire de Byzance. Mais, à la différence des Bulgares, le gouvernement du roi Georges semble plus préoccupé par la situation financière et économique du royaume grec que par la lutte pour libérer la Macédoine ${ }^{5}$. Par ailleurs, les dirigeants grecs, tout comme l'opinion 
publique, sont plus sensibles aux événements dramatiques qui se déroulent en Crète, où les chrétiens sont massacrés par les musulmans ${ }^{6}$.

En 1894, à Athènes, un groupe de jeunes officiers, dont Pavlos Melas, fonde la Société Nationale ou "Ethniki Etairia"'. Ils se proposent d'éveiller un sentiment national grec parmi les chrétiens de l'autre côté du mont Olympe ${ }^{8}$. La majorité des membres de cette association secrète est formée par des sous-officiers ou officiers de l'armée grecque qui ont la ferme intention d'organiser des expéditions armées en Macédoine.

11 Les mouvements grec et bulgare ont un point en commun : chacun bénéficie du support conséquent de la part de chacune des deux puissantes églises orthodoxes, le Patriarcat oeucuménique et l'Exarchat bulgare. Pendant des siècles, les Grecs ont joui d'une situation privilégiée dans les trois vilayets grâce à l'influence du patriarche installé à Constantinople. Dans l'empire ottoman, où pouvoir spirituel et temporel sont intimement liés, le Patriarcat grec a acquis avec le temps un pouvoir considérable auprès des chrétiens, dans la mesure où il réussit à promouvoir les idées de l'Hellénisme à travers le système d'éducation scolaire et les popes, notamment dans les campagnes. Ainsi, l'usage du slavon fut-il progressivement remplacé par celui du grec durant les services religieux. Cependant, autour de 1900, l'influence du Patriarcat se trouve en déclin constant depuis environ deux décennies en raison du fort développement des structures de l'Exarchat bulgare ${ }^{9}$.

Les deux églises sont devenues ennemies en Macédoine et rivalisent pour obtenir la nomination de tel évêque ou l'ouverture d'écoles supplémentaires. Le patriarche, tout comme l'exarque, demande régulièrement l'aide des gouvernements d'Athènes ou de Sofia qui, grâce à leurs diplomates en poste à Constantinople, peuvent soutenir fermement leurs demandes auprès du Sultan. La rivalité entre les deux églises peut, à l'occasion, se révéler violente : les services religieux sont brutalement interrompus par des membres de la communauté adverse, partisans d'imposer le slavon ou le grec, et les soldats ottomans sont envoyés pour séparer les belligérants qui en sont venus aux mains ${ }^{10}$. L'assignement des cimetières peut aussi être à l'origine de tensions, car les exarchistes refusent désormais d'être enterrés aux côtés des patriarchistes ${ }^{11}$. L'Exarchat possède un avantage sur son rival, celui de rassembler sous sa bannière un peuple unique, les Bulgares, alors que les partisans du patriarche sont en majorité des Grecs, mais peuvent aussi être des Valaques ou des Serbes. Dans ce contexte ambigu, les évêques du Patriarcat ou de l'Exarchat se sentent divisés entre leur foi, le dogme et les aspirations nationales des gouvernements dont ils dépendent financièrement.

13 Le mouvement serbe est le plus ancien, mais aussi le plus faible, car ses moyens sont considérablement limités. Bien que le mouvement ait pris une forme organisée dès 1886, avec la création de la Société de Saint-Sava, dont les aspirations nationales sont similaires à celles des associations bulgare ou grecque, les ambitions politiques de la Serbie à l'égard de la Macédoine sont relativement récentes. C'est seulement après 1878, lorsque la Bosnie et l'Herzégovine sont placées sous l'administration de Vienne, que Belgrade oriente ses vues vers le sud des Balkans. Autre point faible, le gouvernement serbe ne dispose pas de l'appui de l'église ou d'institutions religieuses en Macédoine, où les populations serbes sont assimilées aux Grecs-patriarchistes ${ }^{12}$. Par ailleurs, ni le roi Milan, ni son fils Alexandre au pouvoir après 1893, ne possèdent le charisme nécessaire à la conduite d'une politique nationale agressive. Les ambitions serbes en Macédoine se limitent donc à la partie nord du vilayet de Kosovo et autour de la ville même de Skopje. 

un mouvement politique organisé, caractérisé par un esprit social révolutionnaire. C'est en 1893 que six jeunes intellectuels fondent secrètement l'organisation à Salonique. Leur but est de mettre fin à la domination ottomane et d'établir un régime d'autonomie pour tous les habitants de la Macédoine, quelque soit la nationalité revendiquée. Pour réaliser cet objectif, les fondateurs de l'ORIM appuient leurs revendications sur l'article 23 du congrès de Berlin, selon lequel des réformes en faveur des chrétiens devaient être introduites en Turquie d'Europe.

Formés dans les écoles de l'Exarchat, et tous originaires de Macédoine, ils ont décidé de "se mettre au service de leur pays ». Idéalistes, ils se sentent investis d'une mission d'éducateurs à l'égard des populations. Comme les mouvements de Sofia ou Athènes, leur premier objectif est donc d'éveiller le sens de la conscience nationale parmi ces populations si mélangées, grâce à des discussions, des débats et la diffusion de toute une littérature célébrant les héros de la guerre d'indépendance américaine ou Garibaldi. La personnalité marquante du groupe est Damian Gruev, un instituteur et correcteur d'imprimerie. En 1894-1895, il rencontre Goce Delčev, également instituteur, qui avait été renvoyé l'année précédente de l'académie militaire de Sofia pour ses idées socialistes ${ }^{13}$. Les deux hommes deviendront les figures marquantes de l'ORIM et partagent les mêmes vues: la libération doit se faire à partir de l'intérieur de la Macédoine par une organisation strictement macédonienne.

Les relations ambiguës entre l'ORIM, l'Exarchat et le gouvernement de Sofia sont à souligner, dans la mesure où l'exarque, Mgr. Joseph, jouit d'un prestige considérable, qui se double, malgré lui, d'un pouvoir politique, puisqu'être partisan de l'Exarchat signifie être bulgare ${ }^{14}$. Le gouvernement de Sofia a pour politique d'éveiller progressivement un sentiment national bulgare parmi les paysans des trois vilayets. Mais cette alliance, que le nationalisme bulgare crée ainsi entre l'Eglise et les ambitions du gouvernement, va évoluer à cause de l'ORIM. En effet, les instituteurs, membres de l'ORIM, comme ceux du Comité Suprême, sont nommés en Macédoine par Mgr. Joseph. Or, certains d'entre eux vont devenir hostiles au clergé en raison de leurs opinions socialistes et anti-cléricales. Par ailleurs, les dirigeants de l'ORIM vont tenter d'utiliser les structures de l'Exarchat (écoles, couvents) à des fins politiques. Progressivement, le système d'éducation scolaire va devenir un instrument utilisé par le mouvement national bulgare (le Comité Suprême) ou macédonien (l'ORIM). Par conséquent, des tensions graves vont surgir entre l'exarque et les dirigeants de l'ORIM ou bulgares, car Mgr. Joseph refusera toujours de faire passer leurs intérêts avant ceux de son église.

\section{Les activités politiques en Macédoine}

Parmi les quatre mouvements de libération, l'ORIM est le seul qui réussit à mettre en place des structures permanentes à travers l'ensemble des trois vilayets entre 1893 et 1900. Grâce à un réseau dense de comités révolutionnaires, de groupes de combats, les comitadjis, et une organisation stricte, hiérarchisée, fondée sur le secret, l'ORIM établit une administration clandestine parallèle à celle des Ottomans. L'ORIM s'efforce également d'intervenir aux différents stades de la vie quotidienne pour convaincre les populations de l'existence d'un système national macédonien efficace avec sa poste, ses tribunaux clandestins, un service sanitaire capable de distribuer des médicaments, ses bulletins et même un embryon d'organisation financière.

Balkanologie, Vol. IV, $n^{\circ} 2$ | 2000 

société rurale n'a que très progressivement rejoint les rangs de l'ORIM. Même si la vie des paysans est misérable, l'idée de bouleverser complètement leur quotidien et de se lancer dans une voie inconnue effraie la plupart d'entre eux. Dans le nord du vilayet de Bitola et dans celui du Kosovo, l'ORIM devient plus rapidement populaire, car ses combattants se portent à la défense des paysans régulièrement brutalisés par les Albanais. Il fut aussi plus facile pour l'ORIM d'y installer un réseau, car ces contrées de montagnes, difficiles d'accès, échappent de fait à l'administration ottomane. commun : leur objectif qui est de créer un climat d'insécurité, de troubles permanents pour les intérêts ottomans. Entre 1895 et 1899, des révoltes sporadiques éclatent régulièrement dans les campagnes en suivant le rythme des saisons. Au printemps, des groupes armés (15 à 25 hommes environ) franchissent clandestinement les frontières grecque ou bulgare. Suivant une tactique comparable aux comitadjis de l'ORIMA, ces hommes harcèlent constamment les soldats sans engager de réels combats en raison de leurs effectifs restreints. l'église, aux cotés du pope ou de l'instituteur local pour inciter la population à se dresser contre le "joug" ottoman. Les comitadjis distribuent des armes (fusils, bombes) ainsi que des tracts appelant à la révolte ${ }^{15}$. Par ailleurs, l'ORIM organise plusieurs enlèvements contre rançon pour acheter des armes. En 1897-1899, les autorités ottomanes accusent, à quatre reprises, sans faire de distinction, les Bulgares et l'ORIM, et attribuent des motifs politiques à ces actes ${ }^{16}$. En juin 1899, l'enlèvement du directeur des mines d'Isvoro, un Français, Louis Chevalier, près de Serres (vilayet de Salonique) est un succès pour les comitadjis. Louis Chevalier est libéré contre la somme de 15000 livres turques à la suite de négociations longues et compliquées, car ni la société de Kassandra, ni les autorités ottomanes n'entendaient verser la rançon ${ }^{17}$. réseau similaire à celui de l'ORIM. Leurs interventions armées en Macédoine sont, justifient-ils, la réponse à la conduite brutale des Turcs à l'égard de leurs "frères hellènes" qu'ils se doivent de protéger. La plupart de ces groupes armés sont placés sous les ordres d'officiers grecs ou bulgares. Certains d'entre eux ont même demandé un congé, ce qui présente l'avantage de dégager leur gouvernement respectif de toute responsabilité officielle. Vers le mois d'octobre, les partisans suspendent leurs activités, repassent la frontière ou regagnent leurs villages. Les hivers sont en général calmes. Selon les rapports consulaires, il aurait d'ailleurs été impossible aux comitadjis de se maintenir dans les montagnes en raison des rigueurs du climat.

22 À partir de 1895 , on assiste à une escalade de la violence, dans la mesure où les autorités ottomanes ne parviennent pas à enrayer le principe de ces révoltes saisonnières. Chaque année, les Turcs ordonnent des arrestations massives qui engendrent de multiples incidents. Sous le prétexte de fouiller les maisons pour chercher des armes ou des partisans, les soldats pillent et maltraitent les chrétiens qui, par conséquent, vont être d'autant plus sensibles aux discours nationalistes et vengeurs, bulgare, grec ou serbe.

Pendant l'été 1895, les grandes puissances suivent avec attention les expéditions armées organisées par le Comité bulgare de Sofia. En juin-juillet, plusieurs bandes sont envoyées en Macédoine. L'une d'entre elles, supervisée par un ancien officier de 
l'armée bulgare, Boris Sarafov, attaque même, puis incendie, la petite ville de Melnik (au nord du vilayet de Salonique) ${ }^{18}$. En fait, les chefs du Comité Suprême placent tous leurs espoirs dans la diplomatie européenne, dont ils attendent un nouvel examen du statut de la Macédoine en l'associant aux événements d'Arménie, où les chrétiens sont au même moment massacrés par les Turcs. Grâce à la presse, ils essayent d'utiliser le mouvement qui se manifeste en leur faveur dans l'opinion européenne pour amener les puissances à négocier de nouveau avec le gouvernement ottoman. C'est un échec complet, car les diplomates décident d'ignorer la question de Macédoine.

Dans le même esprit, l'été suivant, en 1896, les Grecs de “l'Ethniki Etairia” envoient plusieurs bandes armées dans le vilayet de Salonique ${ }^{19}$. Pendant deux mois, les partisans grecs se battent contre les Turcs, obligeant ces derniers à maintenir un nombre important de troupes en état d'alerte, tout en sachant parfaitement que les soldats ottomans sont irrégulièrement payés et souvent sous équipés ${ }^{20}$. Les bandes font étape dans les monastères du Patriarcat qui, à l'occasion, leur servent aussi de refuge pour échapper aux soldats. En 1897, les armées grecques sont battues de façon humiliante par les Turcs et les activités des bandes grecques en Macédoine sont suspendues ${ }^{21}$. Néanmoins, à Athènes, les cercles intellectuels et militaires poursuivent leurs activités : réunions, discours, pétitions, appels à la défense de la cause grecque en Macédoine.

S'il n'exista aucun accord, ou même de contact, entre les organisations grecque et bulgare, l'ORIM établit des liens avec le Comité Suprême. Ces relations se sont d'autant plus facilement développées que de nombreux instituteurs de l'ORIM ont fait leurs études ou ont vécu à Sofia.

En 1894 et 1895, les chefs de l'ORIM, Goce Delčev et Damian Gruev, se rendent à plusieurs reprises à Sofia et rencontrent ceux du Comité Suprême. En 1896, Delčev organise plusieurs points de passage clandestins le long de la frontière bulgare pour faire entrer des armes, des tracts et des manuels militaires en Macédoine. En février, il rencontre le président du Comité Suprême, le général Danail Nikolaev ${ }^{22}$. L'atmosphère tendue dans laquelle se déroule l'entretien illustre parfaitement la différence de mentalités entre "instituteurs-révolutionnaires" et "militaires" qui séparera, puis opposera, les deux organisations. Défendre une insurrection "de paysans" apparaîtra toujours comme un projet fantaisiste et ridicule aux yeux des militaires du Comité Suprême. Néanmoins, à la fin de l'année 1896, l'ORIM envoie deux représentants permanents, Goce Delčev et Gjorče Petrov, à Sofia pour maintenir les relations avec les milieux politico-financiers de l'émigration macédonienne susceptibles d'être favorables à leur cause.

Entre 1899 et 1901, les deux mouvements se rapprochent, grâce à l'entente qui existe entre Delčev, Petrov et Boris Sarafov, d'origine macédonienne, qui préside alors le Comité Suprême. Des accords sont signés, prévoyant des opérations militaires conjointes. Si les Bulgares disposent des fonds et du support militaire, l'ORIM, en revanche, contrôle "le terrain" en Macédoine, grâce à son réseau de comités révolutionnaires. Néanmoins, les relations entre les "Bulgares" et les "Macédoniens" restent ambiguës en raison des divergences profondes qui subsistent, quant au statut de la future Macédoine libérée: autonomie, voir indépendance pour l'ORIM; intégration pour les Bulgares. Jusqu'en 1912, ces relations évolueront, mais les chefs de l'ORIM s'efforceront toujours d'éviter la rupture complète. en 1895 et 1896. L'initiative est venue des dirigeants du Comité Suprême de Sofia qui, à 
deux reprises, se rendent à Belgrade. Les diplomates européens interprètent ces entrevues comme une alliance possible entre les peuples slaves contre les $\operatorname{Turcs}^{23}$. Karavelov, un des chefs envoyés par Sofia, rencontre plusieurs politiciens serbes défendant les ambitions de Belgrade en "Vieille Serbie", c'est-à-dire le territoire correspondant plus ou moins au vilayet de Kosovo ${ }^{24}$. Néanmoins, ces conversations ne dépassent pas l'échange de vues, car, au même moment, le gouvernement de Belgrade espère qu'en ne soutenant pas les prétentions du Comité Suprême, il obtiendra du Sultan des avantages pour les Serbes de Macédoine, notamment la nomination d'évêques serbes dans le vilayet du Kosovo. De fait, l'année suivante, en 1897, un Irade (décret) autorise officiellement l'ouverture d'écoles serbes dans le vilayet de Salonique ${ }^{25}$.

Il faut également mentionner les négociations entre les Serbes et les Grecs qui, à deux reprises, en 1890-1891 et 1899, vont essayer de parvenir à une entente. Belgrade cherche alors à obtenir des avantages en matière scolaire du Patriarche pour les Serbes de Macédoine. Mais, ce dernier refuse, soucieux de préserver sa complète autorité religieuse dans les trois vilayets. Les conversations évoluent ensuite dans un sens plus politique, avec la tentative de délimiter deux zones d'influence respectives, mais le point d'achoppement reste la ligne de partage du vilayet de Bitola. En 1899, les négociations sont rompues après la visite à Athènes de Miličević, le secrétaire du roi Alexandre ${ }^{26}$.

\section{L'orientation terroriste après 1900 : un choix de l'ORIM}

Vers 1900, le caractère du combat de l'ORIM se transforme, et le mouvement devient terroriste. Ses dirigeants adoptent la politique du pire, ils choisissent de provoquer les Ottomans pour susciter de leur part une réaction brutale, qui entraînera, estiment-ils, l'intervention des puissances ${ }^{27}$. Cette politique extrême est également utilisée par les Arméniens à la même époque.

31 L'ORIM crée donc les tchétas, surnommées par la suite "compagnie de la mort". Ce sont des unités d'élite de sept à dix hommes, prêts à mourir au combat et souvent déjà condamnés par la justice ottomane. Les tchétas sont chargées des attentats et de la destruction des moyens de communication (lignes de télégraphe et de téléphone, ponts, lignes de chemin de fer). En 1901-1902, la situation générale se dégrade sensiblement, car les comitadjis maintiennent un climat de troubles permanents à travers les campagnes, ce à quoi les soldats ottomans répondent en brutalisant les chrétiens, et l'ORIM ordonne de nouveaux attentats et enlèvements. En septembre 1901, la missionnaire américaine Ellen Stone est kidnappée et reste captive de l'ORIM durant six mois. L'affaire fait sensation dans la presse européenne et américaine ${ }^{28}$.

Dans les villes règne une atmosphère tendue aux aspects parfois dramatiques. Des cadavres sont découverts au beau milieu des rues. Les meurtres à caractère politique se multiplient, dirigés essentiellement contre ceux qui représentent les propagandes rivales, directeurs d'écoles, prêtres, agents subalternes des consulats balkaniques ${ }^{29}$. Le meurtre peut aussi être la conséquence d'un échange d'arguments entre partisans de la cause bulgare, grecque ou serbe. Le vice-consul austro-hongrois, August Kral, voit dans l'aggravation des tensions entre les différentes communautés vivant à Bitola le résultat des progrès réalisés en Macédoine par les "propagandes" des différents États balkaniques ${ }^{30}$. 

d'attentats dans le port de Salonique. Un groupe d'anarchistes, "les Bateliers" font exploser tour à tour un navire français, le vapeur "Guadalquivir", les conduites de gaz et d'éclairage urbain, ainsi que le bâtiment de la Banque Impériale Ottomane. "Les Bateliers", tous très jeunes, sont prêts à mourir pour donner à leur sacrifice une portée symbolique. Salonique est en état de choc pendant deux jours et une centaine de personnes sont tuées au cours des fusillades entre les anarchistes et la police. Trois mois plus tard, le 2 août 1903 (Saint Elie), l'ORIM déclenche une insurrection générale qui, à partir du vilayet de Bitola, s'étend au reste de la Macédoine. Les affrontements entre les comitadjis et les soldats durent trois mois. Si la révolte se solde par un échec militaire, car les forces de l'ORIM sont écrasées par les armées du sultan, les chefs macédoniens remportent néanmoins un succès diplomatique, car les grandes puissances imposent un plan de réformes au sultan Abdul Hamid II. Vienne et Saint Petersbourg, alertées par la dégradation de la situation, décident d'agir de concert ${ }^{31}$. Les accords de Mürzsteg signés en novembre 1903, prévoient des mesures pour rétablir l'ordre et la sécurité, ainsi que l'introduction d'un plan de réformes en faveur des chrétiens de Macédoine. Les trois vilayets sont divisés en cinq secteurs, placés chacun sous le contrôle de l'une des grandes puissances ${ }^{32}$.

L'orientation terroriste de l'ORIM a des conséquences sur la conduite des autres mouvements de libération. En 1904, l'ORIM est affaiblie: le réseau des comités révolutionnaires est en partie détruit, plusieurs chefs ont été tués dans les combats. La défaite d'Ilinden ébranle l'organisation dans ses fondements mêmes, des tensions surgissent quant à la nouvelle ligne politique à définir. Par conséquent, dès le printemps 1904, la Macédoine est de nouveau dévastée par des groupes armés venus de Grèce, de Bulgarie, de Serbie et même de Roumanie qui vont profiter de l'affaiblissement de l'ORIM.

Par ailleurs, le programme de Mürzsteg lui-même est certainement à l'origine de ces nouveaux troubles. Pour les grandes puissances, ces accords ne pouvaient évidemment pas constituer une garantie de complète stabilité en Macédoine, mais apparaissaient comme une solution préférable au départ des Turcs de la région et aux désordres inévitables qui s'en suivraient. Le texte s'applique à l'ensemble des chrétiens et élimine, de ce fait, les revendications nationales grecques, bulgares ou serbes. Cependant, ces mêmes chrétiens interprètent, au contraire, ce texte comme la garantie d'un soutien des puissances et vont l'utiliser pour justifier leurs actions et ingérences en Macédoine.

L'ambiguïté et le manque de précision qui caractérisent le texte des accords de Mürzsteg contribuent à aggraver la situation. L'article 3 stipule une nouvelle délimitation administrative en "vue d'un groupement plus régulier des différentes nationalités", dès que la sécurité sera rétablie ${ }^{33}$. Or, la notion de nationalité dans l'Empire ottoman est presque inexistante, dans la mesure où les populations s'identifient d'abord en fonction de leur appartenance religieuse. Ainsi, l'article 3 ouvre la porte à une nouvelle forme de conflit national-religieux extrêmement violent. Par exemple, les bandes armées grecques forcent les villages de l'Exarchat à rejoindre les rangs du Patriarcat et ainsi à les assimiler aux Grecs. Les populations sont brutalisées et "passent" plusieurs fois d'une Église à l'autre ${ }^{34}$.

En 1904-1905, les Grecs installent rapidement en Macédoine un réseau semblable à celui de l'ORIM. Utilisant leur consulat de Salonique comme "quartier général", ils font passer en Macédoine des groupes armés recrutés en Grèce ou en Crète ${ }^{35}$. Ces partisans 
grecs se battent contre ceux venus de Bulgarie ou de l'ORIM bien plus que contre les Turcs. Les andartes grecs sont soutenus par le clergé du Patriarcat qui souhaite reprendre le terrain occupé (paroisses, évêchés) par l'Exarchat. Au sud de Bitola, l'évêque de Kastoria, Mgr. Karavangelis, prend même une part active à la lutte et tient des discours virulents défendant la cause de l'Hellénisme : « vous êtes Grecs, mais les Slaves arrivèrent et vous êtes devenus des esclaves $»^{36}$. Les Grecs remportent des succès dans le vilayet de Salonique et au sud de celui de Bitola.

La même année, au nord de la Macédoine, les Serbes organisent des incursions à travers le vilayet de Kosovo. Comme les Grecs, ils se servent de leurs consulats pour envoyer et recevoir des instructions, des armes ou des hommes. En 1904, les populations serbes se sentent "exclues" du Patriarcat, elles ne peuvent se tourner vers l'Exarchat ou les groupes bulgares, ni vers l'ORIM, divisée à leur propos. Les Serbes sont donc d'autant plus attentifs au mouvement dirigé depuis Belgrade, qui a pour ambition le rattachement de la "Vieille Serbie" au royaume de Pierre Karadjordjevićc ${ }^{37}$. Des instituteurs, des popes de villages, bien que relevant officiellement du Patriarcat, se déclarent "Serbes", utilisant ainsi un concept national pour marquer une distance vis à vis de "leur Église".

Désormais, l'objectif de ces groupes grecs, bulgares, serbes ou de l'ORIM ne semble plus être la libération de la Macédoine du "joug" ottoman, mais bien la défaite des factions rivales; en d'autres termes, il s'agit d'une guerre entre les éléments chrétiens. Si les mouvements grec et serbe ne concluent aucun accord, il existe entre eux une entente de fait contre tout ce qui représente les Bulgares. Les activités des bandes grecques s'achèvent là où commencent celles des Serbes ${ }^{38}$.

En 1905, un autre "groupe national" fait irruption sur la scène macédonienne, les Roumains, mais leurs activités restent limitées, dans la mesure où il n'existe pas de frontière commune entre la Roumanie et les vilayets macédoniens. Contrairement aux Grecs et aux Bulgares, le roi Charles n'émet aucune prétention territoriale, mais il entend faire reconnaître la réalité de la communauté aroumaine par le Sultan ${ }^{39}$. En outre, les éléments koutzo-valaques représentent à peine $3 \%$ de la population des trois vilayets. Cependant, les bandes aroumaines vont se battre dans le vilayet de Bitola contre les Grecs aux côtés de l'ORIM. Bien que les activités roumaines restent marginales par rapport à celles des Grecs ou de l'ORIM, elles n'en constituent pas moins un facteur de troubles supplémentaires, qui contribue à la détérioration générale de la situation en Macédoine.

41 Jusqu'à la Révolution Jeune Turque de juillet 1908, la situation continue de s'aggraver, aucune amélioration n'apparaît, et le Sultan utilise ce climat de troubles à son profit, dressant les minorités chrétiennes tour à tour les unes contre les autres. Il laisse d'abord les bandes grecques se battre contre les comitadjis autour de Bitola, là où les structures de l'ORIM étaient le mieux implantées. Puis, il laisse les groupes grecs agir à leur guise contre les Bulgares et l'ORIM autour de Skopje. À partir de 1905, il ne prend aucune mesure contre les partisans valaques dans leur lutte contre les Grecs, devenus trop "puissants" à ses yeux, ni contre les Albanais qui mettent à sac les villages serbes du vilayet de Kosovo ${ }^{40}$. 


\section{Conclusion}

\section{NOTES}

1. Les autres principaux territoires étaient constitués par l'Albanie (les deux vilayets de Janina et Shkodër) et la Thrace (le vilayet d'Andrinople).

2. En 1878, la Macédoine fut brièvement incluse durant trois mois dans "la grande Bulgarie" entre le traité de San Stefano (mars) et celui de Berlin (juin). Au Congrès de Berlin (13 juin-13 juillet 1878), les grandes puissances (Russie, Autriche-Hongrie, Allemagne, Italie, GrandeBretagne, France) imposent la révision du traité de San Stefano (3 mars 1878) qui avait mis fin à la guerre russo-turque de 1877-78. La Serbie, le Monténégro, la Roumanie deviennent indépendants. La Bulgarie obtient le statut de principauté vassale du gouvernement ottoman. L'Autriche-Hongrie a désormais le droit d'occuper militairement la Bosnie et l'Herzégovine ainsi que d'entretenir des garnisons dans le sandjak de Novi-Pazar qui fait partie du vilayet de Kosovo. Les trois vilayets macédoniens sont simplement rendus à l'empire ottoman.

3. Vers 1900, Salonique comptait environ 150000 habitants. Dumont (Paul), « Une organisation socialiste ottomane : la fédération ouvrière de Salonique 1908-1912 ", Etudes Balkaniques, (1), 1975.

4. Lange-Akhund (Nadine), The Macedonian Question 1893-1908. From Western Sources, Boulder : Columbia University Press, 1998, p. 49. En 1885, la province ottomane de Roumélie Orientale se souleva contre le sultan et obtint son rattachement à la Bulgarie.

5. La vie politique était dominée par une grande instabilité ministérielle. Entre 1893 et 1910, vingt gouvernements se succédèrent. 
6. En Crète, possession ottomane, la situation était troublée depuis le début des années 1820. Les Crétois chrétiens demandaient leur rattachement à la Grèce. Les incidents entre les deux communautés musulmane et chrétienne étaient permanents.

7. Pavlos Melas était le gendre du ministre des affaires étrangères Dragoumis. Tué dans un combat contre les soldats ottomans en Macédoine en octobre 1904, il devint un héros et un martyr de la cause grecque en Macédoine.

8. “L'Ethniki Etairia" était aussi présente et active en Crète. Vakalopoulos (K.A.), Modern History of Macedonia, Thessaloniki, 1988, p. 201.

9. En 1870, le sultan autorisa l'établissement de l'Exarchat bulgare qui jouissait d'une autonomie vis à vis du Patriarcat. Selon l'article 10 du firman (décret), si les deux tiers des habitants d'une éparchie réclamaient leur rattachement à l'Exarchat, la communauté pouvait rejoindre les rangs de l'église bulgare. En 1872, le patriarche excommunia l'exarque.

10. HHsta Arch. Amb, vol 491, Wass à Calice, Salonique 24 décembre 1894. AE CP Turquie, vol 23, Rousseau à Hanotaux, Belgrade 3 mai 1898.

11. AE CP Turquie, vol 22, Ledoulx à Hanotaux, Monastir 27 décembre 1897.

12. Le patriarcat serbe de Peć a été aboli en 1766.

13. Les deux hommes sont contemporains. Damian Gruev : 1871-1906, Goce Delčev : 1872-1903.

14. Mgr. Joseph est né en 1840 à Kalofer (Bulgarie) et décédé en 1915 à Sofia. Von Mach (R.), The Bulgarian Exarchate, its history and the exents of authority in Turkey, London, 1905, pp. 20-21.

15. Perry (Duncan M.), The politic of Terror. The Macedonian Liberation Movement 1893-1908, Durham : Duke University Press, 1988, p. 152.

16. HHsta PA 38 Monastir, vol 389, Kral à Goluchowski, 23 septembre 1898.

17. $A E$ Arch Amb CC Salonique, vol 1019, Ledoulx à Constans 16 juin 1899, Gaulier à Steeg 12 et 13 juin 1899.

18. AE CP Arch Amb Macédoine, vol 138, Naggiar à Cambon, Salonique 26 juillet 1895. AE CP Turquie, vol 522, Cambon à Hanotaux, Pera 29 juillet 1895.

19. AE Arch Amb Macédoine, vol 139, Naggiar à Cambon, Salonique 23 juillet 1896.

20. AE CP Turquie, vol 21, Ledoulx à Hanotaux, Monastir 20 août 1896. HHsta PA 38 Monastir, vol 389, von Kwiatkowski à Goluchowski, 28 juillet 1896.

21. La guerre gréco-turque de 1897 fut brève (avril-mai) et se termina par la défaite de l'armée grecque à la bataille de Larissa (17 mai 1897).

22. Mac Dermott (Mercia), Freedom or Death, the life of Gotse Delchev, London : Journeyman Press, 1978, p. 132. Poljanski (Hristo Andonov), Documents on the struggle of the Macedonian people for independence and a nation state, University of Cyril and Methodius, 1985 vol 1, p. 357.

23. $A E C P$ Serbie, vol 16, Patrimonio à Hanotaux, Belgrade 23 juin 1895.

24. $A E C P$ Serbie, vol 16, Patrimonio à Hanotaux, Belgrade 16 septembre 1895. Des contacts furent établis avec Nikola Pašić (1845-1926) du parti radical et avec Ristić du parti libéral.

25. AE Arch Amb CC Macédoine, vol 139, Veillet-Dufrèche à Cambon, Salonique 26 avril 1897. Le décret fut proclamé avant le début de la guerre gréco-turque de 1897. Le Sultan pensait ainsi se garantir la neutralité de la Serbie.

26. Langer (William Leonard), The diplomacy of imperialism 1890-1902, New York, 1951, pp. 308-310. Les Serbes demandaient le vilayet de Bitola jusqu'à Kastoria et Florina. Driault (Edouard), Lhéritier (Michel), Histoire diplomatique de la Grèce de 1821 à nos jours, Paris : PUF, 1926, tome 4, pp. 482-483.

27. Selon un des chefs macédoniens, G. Petrov, le choix de recourir à "des méthodes terroristes" n'a pas fait l'objet d'une décision formelle, mais lors du congrès annuel de 1901, le pas était franchi. Par ailleurs, Delčev partageait les idées des Libertaires, selon lesquelles il fallait créer une insécurité permanente pour les intérêts des grandes puissances afin d'ébranler l'empire ottoman. Bozhinov (Voin), La Macédoine: recueil de documents et matériaux, Sofia: Académie bulgare des Sciences, 1980, pp. 548-549. 
28. Miss Stone fut relâchée contre 14000 livres turques. Après sa libération, elle s'attacha à la cause de l'ORIM et donna plusieurs conférences aux Etats-Unis. Sherman (Laura Beth), Fires on the Mountain, New York : Columbia University Press, 1980.

29. AE CP Turquie, vol 24, Ledoulx à Delcassé, Monastir 8 février 1899.

30. HHsta PA 38 Monastir, vol 390, Kral à Goluchowski, 5 février 1899 et 10 novembre 1900.

31. L'Autriche-Hongrie et la Russie ont pris l'initiative d'agir dans les Balkans, soucieuses d'y préserver leur position exclusive.

32. Autriche-Hongrie, Russie, France, Grande-Bretagne et Italie. L'Allemagne refusa de se charger d'un secteur. Les accords de Mürzsteg comprenaient neuf points.

33. Article 3 : «Aussitôt qu'un apaisement du pays sera constaté, demander au gouvernement ottoman une modification dans la délimitation des unités administratives en vue d'un groupement plus régulier des différentes nationalités ».

34. AE CP Turquie, vol 54, Steeg à Delcassé, Salonique 4 octobre 1907. «Le conflit des nationalités en Macédoine se présente comme une lutte d'églises bien plus que comme une lutte de races ", commente Steeg.

35. AE CP Turquie, vol 45, Ormesson à Delcassé, Athènes 4 mai 1905. AE CP Turquie, vol 48, Maurouard à Rouvier, La Canée 13 mars 1906. Le recrutement des Crétois posa rapidement une difficulté: ils haïssaient tellement les musulmans qu'ils ne comprenaient les raisons qui poussaient les Grecs à combattre d'autres chrétiens (Bulgares ou Serbes).

36. Vakalopoulos (K.A.), op.cit., p. 212. HHsta PA 38 Monastir, vol 394, Kral à Goluchowski, 16 avril 1904. Dakin (Douglas), The Greek struggle in Macedonia, Thessaloniki : Institute for Balkan Studies, 1966, p. 126.

37. Le roi Pierre I prit le pouvoir en juin 1903, après l'assassinat du roi Alexandre et de sa femme Draga.

38. AE CP Turquie, vol 52, Boulinière à Pichon, Athènes 10 mai 1907.

39. En 1905, les Aroumains de Macédoine sont reconnus en tant que communauté civile au sein de l'empire ottoman tout en continuant à dépendre du Patriarcat pour la nomination de leurs évêques.

40. Chiclet (Christophe), Lory (Bernard), éds., La République de Macédoine, Paris : L'Harmattan, 1998, pp. 39-64.

41. Mac Dermott (Mercia), op.cit., p. 227.

42. $A E C P$ Turquie, vol 45, Choublier à Rouvier, Uskub 8 juin 1905. Durant un entretien de trois heures, Gruev s'efforce de convaincre Simić, un des chefs politiques serbes, de la réalité politique de l'ORIM, sans laquelle les gouvernements de Belgrade ou Sofia ne pourront résoudre la question macédonienne.

43. AE CP Turquie, vol 48, Gauthier à Constans, Monastir 1 avril 1906.

\section{INDEX}

Mots-clés : Macédoine 